ISSN 0103-5150

Fisioter. Mov., Curitiba, v. 26, n. 3, p. 481-489, jul./set. 2013

Licenciado sob uma Licença Creative Commons

\title{
Riscos de distúrbios osteomusculares em punhos de trabalhadores de uma indústria de pescados
}

\author{
Risks of musculoskeletal disorders in wrist \\ for workers of a fish industry
}

\section{Bruno Maia de Guimarães ${ }^{[a]}$, Leonardo Soares de Azevedo ${ }^{[b]}$}

[a] Doutorando em Design e Mestre em Design pela Universidade Federal de Pernambuco (UFPE), Recife, PE - Brasil, e-mail: bmguimaraes@hotmail.com

[b] Especialista em Ergonomia pela Universidade Federal de Pernambuco (UFPE), Recife, PE - Brasil, e-mail: leoaz@uol.com.br

\section{Resumo}

Introdução: Os distúrbios osteomusculares relacionadas ao trabalho são patologias muito frequentes no meio industrial, principalmente quando os trabalhadores se encontram expostos a fatores de risco como, por exemplo, posturas inadequadas, repetitividade, uso de força excessiva e a exposição a vibrações. Objetivo: Avaliar o risco de distúrbios osteomusculares nos punhos de trabalhadores de uma indústria de pescados da cidade de Recife (PE). Metodologia: Utilizou-se metodologia qualitativa baseada no método Strain Index (SI), o qual resulta em uma pontuação numérica que aponta a probabilidade de desenvolvimento de distúrbios osteomusculares no punho. 0 índice é baseado na pontuação do SI, representado pela interação multiplicativa entre as variáveis da tarefa: intensidade do esforço, duração do esforço, frequência do esforço, postura da mão e punho, ritmo de trabalho e duração diária da tarefa. Resultados: 0 valor encontrado foi de SI = 9 para ambos os punhos, classificando-o como uma atividade com alto risco para o desenvolvimento de distúrbios osteomusculares nos punhos dos trabalhadores. Conclusão: A indicação da presença dos riscos desses distúrbios nos punhos durante a realização da atividade de trabalho foi determinada pela pontuação do SI, sugerindo a necessidade de intervenção ergonômica.

Palavras-chave: Fatores de risco. Saúde do trabalhador. Distúrbios osteomusculares relacionados ao trabalho. Ergonomia. 


\section{Abstract}

Introduction: Cumulative trauma disorders are very common diseases in industry, especially when workers are exposed to risk factors such as, for example, inappropriate postures, repetitive, excessive use of force and vibration exposure. Objective: To assess the risk of musculoskeletal disorders in the wrists of industrial workers caught in the city of Recife, Pernambuco State, Brazil. Methodology: We used a qualitative methodology based on the method Strain Index (SI) which results in a numerical score that indicates the risk of developing musculoskeletal disorders in the wrist. The index is based on the SI score is represented by the multiplicative interaction between the task variables: intensity of exercise, exercise duration, frequency of stress, the hand and wrist posture, pace of work and daily duration of the task. Results: The value found was SI = 9 for both fists, classifying it as a risky activity for the development of musculoskeletal disorders in the wrists of workers. Conclusion: The indication of the presence of the risks of these disorders in the wrists during the course of work activity was determined by the SI score, pointing to the need for ergonomic intervention.

Keywords: Risk factors. Occupational health. Cumulative trauma disorder. Ergonomics.

\section{Introdução}

A relevância de estudos que visam à prevenção de lesões musculoesqueléticas em situações ocupacionais deve-se ao fato de essas doenças gerarem consequências sociais e econômicas para o Estado, empresas e indivíduos. Para a empresa, significa a redução no número de homens/horas trabalhadas, devido à ausência no trabalho por períodos de tempo consideráveis, o que provoca uma perda na produção e na qualidade do serviço; para o Estado, as despesas recaem sobre o Instituto Nacional do Seguro Social (INSS) e decorrem do pagamento de benefícios previdenciários, tratamento e reabilitação (1).

Segundo dados da Previdência Social, observa-se que em 2002 foram registrados aproximadamente 22 mil casos de LER/DORT; em 2003 foram registrados 23 mil casos e, em 2004, cerca de 27 mil casos. Ainda segundo o Ministério da Previdência Social, os gastos em 2005 com o pagamento de benefícios decorrentes de acidentes de trabalho atingiram a quantia de R\$ 9,8 bilhões. Ainda assim, verifica-se que é consensual a opinião dos que atuam na área, de que há uma subnotificação de cerca de $80 \%$ dos acidentes do trabalho, boa parte deles contabilizados entre os segurados da Previdência Social que recebem benefícios não acidentários (2).

Devido ao alto custo no tratamento das LER/DORT e o grande número de subnotificações, o decreto n. 6.042 da Previdência Social entrou em vigor em abril de 2007 e regulamenta as mudanças na caracterização das doenças e acidentes relacionados ao trabalho pelo novo sistema de nexo técnico epidemiológico (NTEP). Assim, as doenças ocupacionais passaram a ser caracterizadas tecnicamente pela perícia médica do INSS, mediante a identificação do nexo causal entre o trabalho e a doença, quando se verifica a relação entre a atividade da empresa, identificada pela Classificação Nacional de Atividade Empresarial (CNAE), e a doença ou sequela que motivou a incapacidade, identificada pelo Código Internacional de Doenças (CID) (3).

A partir desse momento, o número de notificações de casos de LER/DORT entre os trabalhadores tende a crescer consideravelmente. Isso porque grande quantidade de casos era subnotificada anteriormente e por causa da notificação de casos nos quais existe a relação do CNAE da empresa com o CID do distúrbio musculoesquelético do trabalhador, mas ele não exerce atividade que poderia causar a lesão (3). Em corroboração, dados do INSS de 2007 mostram que houve um aumento de $148 \%$ em relação ao ano anterior, na concessão de auxílios-doença de natureza acidentária, nos quais fazem parte as LER/DORT (4).

As lesões musculoesqueléticas relacionadas ao trabalho são patologias muito frequentes em meio industrial, principalmente quando os trabalhadores se encontram expostos a fatores de risco como, por exemplo, posturas extremas, repetitividade gestual, aplicações de força com a mão ou dedos e a exposição a vibrações $(5,6)$.

Em pesquisas com trabalhadores de processamento de peixes de indústrias tailandesas, foram encontradas prevalências de 30,9\% de doenças do 
ombro e pescoço, $15 \%$ de epicondilite e $14,5 \%$ de síndrome do túnel do carpo (7).

Os fatores biomecânicos que mais contribuem na origem da LER/DORT são a força, a repetitividade, a velocidade dos movimentos e a duração da atividade. Dessa forma, as posturas desconfortáveis de trabalho, limitadas, assimétricas, repetidas e/ou prolongadas, os movimentos extremos e/ou repetitivos, e a utilização de força excessiva podem causar sobrecarga nos tecidos e exceder seus limites de estresse, causando lesões teciduais devido a esforços inadequados (8). Além dos fatores biomecânicos, existem os organizacionais (pausas, ritmos, sazonalidade da produção, estruturas de horários, métodos impróprios de trabalho, forma da produção), fatores individuais (gravidez, doenças crônicas, sexo, hereditariedade, prática de esportes) e fatores psicossocias (satisfação no trabalho, relacionamento com os colegas de trabalho, ansiedade e expectativa individual) (9).

Assim, percebe-se a necessidade de estratégias preventivas, sendo a fisioterapia do trabalho um dos recursos para o desenvolvimento de programas preventivos nas empresas. A fisioterapia tem como objetivo de atuação não só o resgate da saúde do trabalhador, mas também sua manutenção e prevenção, evitando o surgimento das LER/DORT. Considerando a relevância da prevenção de lesões/disfunções, o objeto de interesse da fisioterapia do trabalho são os fatores de risco presentes no ambiente de trabalho que podem promover ou facilitar a ocorrência destas. A análise desses fatores é o primeiro passo para que as intervenções, tanto preventivas quanto curativas, na saúde do trabalhador tenham sucesso $(10,11)$.

Tendo em vista a influência dos fatores pessoais, biomecânicos, organizacionais e psicossociais relacionados ao trabalho, a avaliação desses fatores é necessária para o estabelecimento da associação entre estes e a possibilidade de surgir e/ou agravar um quadro de sinais e sintomas no trabalhador (12). Com base na identificação dos fatores de risco e de suas características, podem ser tomadas medidas e intervenções ergonômicas e preventivas para a preservação da saúde desses indivíduos (13).

Os fatores de risco podem ser identificados com e emprego de diversas ferramentas ergonômicas, variando de acordo com o tipo de atividade, tipo de risco e realidade observada na organização. A aplicação dessas ferramentas permite classificar as situações de baixo risco a alto risco de desenvolver distúrbios osteomusculares. A maioria desses instrumentos restringe a análise de variáveis biomecânicas, desconsiderando a organização do trabalho e os problemas psicossociais como fator de risco para o aparecimento de LER/DORT $(14,15)$. Dessa forma, o método Strain Index (SI) tem sua importância para a avaliação do risco de distúrbios osteomusculares nos punhos, pois além das variáveis biomecânicas, considera também fatores organizacionais do trabalho (16).

Atualmente, percebe-se a escassez de estudos sobre os riscos de distúrbios osteomusculares em trabalhadores de indústrias de pescados. Dentro desse contexto, este artigo busca avaliar o risco desses distúrbios nos punhos em trabalhadores do setor de filetagem de uma indústria de pescados da cidade de Recife-PE.

\section{Metodologia}

O estudo foi realizado no setor de filetagem de uma empresa de beneficiamento de pescados na cidade de Recife (PE), o qual é composto por dez funcionários, cinco do sexo masculino e cinco do feminino, que têm como função retirar a cabeça do camarão. Todos assinaram o Termo de Consentimento Livre e Esclarecido. 0 presente estudo foi aprovado pelo Comitê de Ética em Pesquisa do Hospital Agamenon Magalhães de Pernambuco e encontra-se de acordo com as normas vigentes.

Foi utilizada a metodologia qualitativa SI, que resulta em uma pontuação numérica correlacionada com o nível de risco de desenvolver distúrbios osteomusculares nos punhos (16). 0 índice é baseado na interação multiplicativa entre as variáveis da tarefa, de acordo com princípios fisiológicos, biomecânicos e epidemiológicos (16). A pontuação do SI representa o produto de seis multiplicadores que correspondem a seis variáveis da tarefa, que são: (a) intensidade do esforço, (b) duração do esforço, (c) frequência do esforço, (d) postura de mão-punho, (e) ritmo de trabalho, e (f) duração diária do trabalho.

A intensidade do esforço é uma estimativa do esforço muscular necessário para executar a tarefa com base na observação do pesquisador, usando descrições verbais que estimam o esforço percebido; essa estimativa é semelhante à usada na escala de Borg (17). As classificações da intensidade de esforço e os respectivos multiplicadores encontram-se na Tabela 1. 
Tabela 1 - Intensidade do esforço de acordo com a escala de Borg (1979)

\begin{tabular}{|c|c|c|c|c|}
\hline Classificação & Observação & Multiplicador & D & $\mathbf{E}$ \\
\hline Leve & Tranquilo (0-2). & 1.0 & & \\
\hline Médio & Percebe-se algum esforço (3). & 3.0 & & \\
\hline Pesado & Esforço nítido; sem expressão facial (4-5). & 6.0 & & \\
\hline Muito Pesado & Esforço nítido; muda a expressão facial (6-7). & 9.0 & & \\
\hline Próximo do Máximo & Usa tronco e membros (8-10). & 13.0 & & \\
\hline
\end{tabular}

Legenda: $\mathrm{D}=$ punho direito; $\mathrm{E}=$ punho esquerdo.

Fonte: Adaptado de Moore e Garg (16).

A duração do esforço físico é caracterizada pela percentagem do tempo que um esforço é aplicado durante o ciclo da atividade. É calculado dividindo a duração de esforço em um ciclo pelo tempo de ciclo multiplicado por 100. 0 tempo de ciclo é calculado dividindo o período de tempo de observação pelo número de esforços realizados.

$\underset{\text { esforço }}{\% \text { Duração do }}=\frac{\begin{array}{c}100 \times \text { duração do esforço } \\ \text { em um ciclo }\end{array}}{\text { Tempo de ciclo }}$

As classificações da duração do esforço e os multiplicadores correspondentes encontram-se na Tabela 2.

Tabela 2 - Duração do esforço

\begin{tabular}{|c|c|c|c|c|}
\hline Classificação & Observação & Multiplicador & D & $\mathrm{E}$ \\
\hline$<10 \%$ do ciclo & & 0.5 & & \\
\hline $10-29 \%$ do ciclo & & 1.0 & & \\
\hline $30-49 \%$ do ciclo & & 1.5 & & \\
\hline $50-79 \%$ do ciclo & & 2.0 & & \\
\hline$>80 \%$ do ciclo & & 3.0 & & \\
\hline
\end{tabular}

Legenda: $\mathrm{D}=$ punho direito; $\mathrm{E}=$ punho esquerdo.

Fonte: Adaptado de Moore e Garg (16).

A frequência do esforço é medida pela quantidade de esforços realizados por minuto. Por exemplo, se a duração do esforço no ciclo for de 20 segundos, então equivale a 3 esforços por minuto. As classificações da frequência do esforço e os respectivos multiplicadores encontram-se na Tabela 3.

Tabela 3 - Frequência do esforço

\begin{tabular}{|c|c|c|c|c|}
\hline Classificação & Observação & Multiplicador & D & $E$ \\
\hline$<4$ por minuto & & 0.5 & & \\
\hline 4-8 por minuto & & 1.0 & & \\
\hline 9-14 por minuto & & 1.5 & & \\
\hline $15-19$ por minuto & & 2.0 & & \\
\hline$>20$ por minuto & & 3.0 & & \\
\hline
\end{tabular}

Legenda: $\mathrm{D}=$ punho direito; $\mathrm{E}=$ punho esquerdo.

Fonte: Adaptado de Moore e Garg (16).

A classificação da postura de mão-punho está relacionada à posição neutra durante a execução da tarefa e pode ser observada na Tabela 4, assim como a sua correlação com os multiplicadores.

0 ritmo do trabalho é a velocidade com que o trabalhador realiza a tarefa. Na Tabela 5 observam-se as classificações da velocidade de trabalho e seus respectivos multiplicadores.

A duração do trabalho por dia é o tempo total em horas que a atividade é desempenhada diariamente. Suas classificações e seus respectivos multiplicadores de acordo com o método SI podem ser observados na Tabela 6.

O valor da pontuação do SI é produto dos multiplicadores das seis variáveis do trabalho. Após determinar essa pontuação, é realizada a classificação quanto ao grau de risco de desenvolver distúrbios musculoesqueléticos nos punhos (Tabela 7). 
Tabela 4 - Postura da mão-punho

\begin{tabular}{|c|c|c|c|c|}
\hline Classificação & Observação & Multiplicador & D & E \\
\hline Muito boa & $\mathrm{DU}=0 \mathrm{a} 10^{\circ}, \mathrm{EXT}=0 \mathrm{a} 10^{\circ}, \mathrm{FLE}=0 \mathrm{a} 5^{\circ}$ & 1.0 & & \\
\hline Boa & $\mathrm{DU}=11 \mathrm{a} 15^{\circ}, \mathrm{EXT}=11 \mathrm{a} 25^{\circ}, \mathrm{FLE}=6 \mathrm{a} 15^{\circ}$ & 1.0 & & \\
\hline Razoável & $\mathrm{DU}=16 \mathrm{a} 20^{\circ}, \mathrm{EXT}=26 \mathrm{a} 40^{\circ}, \mathrm{FLE}=16$ a $30^{\circ}$ & 1.5 & & \\
\hline Ruim & $\mathrm{DU}=21 \mathrm{a} 25^{\circ}, \mathrm{EXT}=41$ a $55^{\circ}, \mathrm{FLE}=31$ a $50^{\circ}$ & 2.0 & & \\
\hline Muito ruim & $\mathrm{DU}>25^{\circ}, \mathrm{EXT}>60^{\circ}, \mathrm{FLE}>50^{\circ}$ & 3.0 & & \\
\hline
\end{tabular}

Legenda: $\mathrm{DU}$ = desvio ulnar; EXT = extensão; FLE = flexão; $\mathrm{D}=$ punho direito; $\mathrm{E}$ = punho esquerdo.

Fonte: Adaptado de Moore e Garg (16).

Tabela 5 - Ritmo do trabalho ou velocidade do trabalho

\begin{tabular}{|c|c|c|c|c|}
\hline Classificação & Observação & Multiplicador & D & $\mathrm{E}$ \\
\hline Muito lento & $\leq 80 \%$ (extremamente relaxado) & 1.0 & & \\
\hline Lento & 81-90\% (faz seu próprio ritmo) & 1.0 & & \\
\hline Razoável & $91-100 \%$ (velocidade normal do movimento) & 1.0 & & \\
\hline Rápido & 101-115\% (apressado, porém acompanha) & 1.5 & & \\
\hline Muito rápido & > 115\% (apressado, não acompanha) & 2.0 & & \\
\hline
\end{tabular}

Legenda: $\mathrm{D}=$ punho direito; $\mathrm{E}=$ punho esquerdo.

Fonte: Adaptado de Moore e Garg (16).

Tabela 6 - Duração do trabalho por dia

\begin{tabular}{lcccc}
\hline Classificação & Observação & Multiplicador & D & E \\
\hline$\leq 1$ & 0.25 & & \\
$1-2$ & 0.5 & & \\
$2-4$ & 0.75 & & \\
$4-8$ & 1.0 & & \\
$>8$ & 1.5 & & \\
\hline
\end{tabular}

Legenda: $\mathrm{D}=$ punho direito; $\mathrm{E}=$ punho esquerdo.

Fonte: Adaptado de Moore e Garg (16).

A coleta de dados foi realizada pela abordagem in loco em três visitas de fisioterapeutas experientes no uso do SI, os quais observaram os funcionários efetivando a retirada da cabeça do camarão. Para a análise, foi realizada observação do registro postural e dos movimentos por meio de fotografia e gravação de vídeo em máquina digital (modelo DSC-S730, Sony). Esses procedimentos foram autorizados pela
Tabela 7 - Classificação do grau de risco

\begin{tabular}{ll}
\hline SI $<3$ & Segura \\
SI entre 3 e 5 & Incerta \\
SI entre 5 e 7 & Algum risco \\
SI $>7$ & Arriscada \\
\hline
\end{tabular}

Fonte: Adaptado de Moore e Garg (16).

empresa e realizados em momentos convenientes, sem que provocassem qualquer alteração ou perturbação na rotina de trabalho dos funcionários.

\section{Resultados}

Na atividade de retirada da cabeça do camarão, os trabalhadores seguram a cabeça da pesca com a mão esquerda e o seu corpo com a mão direita e, em seguida, retiram a cabeça fazendo desvio ulnar de ambos os punhos (v. Figura 1). 

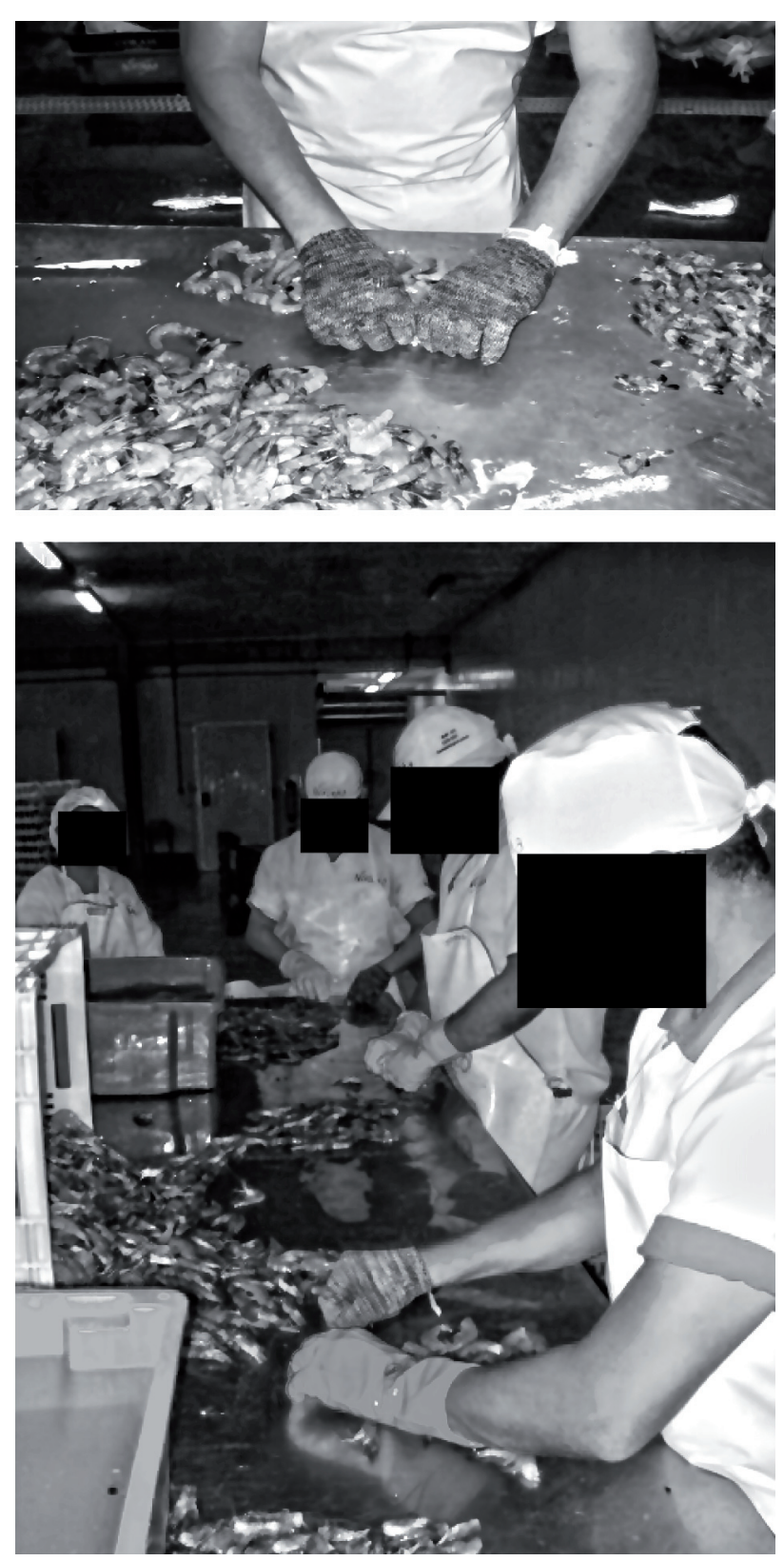

Figuras 1 - Retirada da cabeça do camarão

Fonte: Dados da pesquisa.

Nota: Local de trabalho dos sujeitos da pesquisa. Na ocasião, encontravam-se realizando a atividade de retirada da cabeça do camarão no setor de filetagem da empresa de beneficiamento de pescados.

A intensidade do esforço para a tarefa analisada foi classificada como leve tanto para o punho direito quanto para o esquerdo e corresponde ao multiplicador 1 .

0 tempo médio do ciclo para a retirada da cabeça do camarão foi de 1,7 segundos, sendo o mesmo tempo da duração do esforço realizado pelos trabalhadores para a execução da tarefa, o que corresponde a uma duração de esforço de 100\%. Assim, os valores obtidos correspondem ao multiplicador de valor de 3 para os punhos direito e esquerdo.

0 trabalhador desenvolveu uma frequência média de 35 esforços por minuto durante a retirada da cabeça dos camarões, para ambos os punhos, equivalendo ao valor 3 para o multiplicador.

Após analisar a atividade, as posturas de mãos-punhos direito e esquerdo foram classificadas como muito boa e boa, respectivamente, correspondendo ao valor de 1 do multiplicador para ambos o membros durante a retirada da cabeça do camarão.

A velocidade do trabalho encontrada na atividade foi classificada como razoável, pois os indivíduos realizam numa velocidade normal de movimento, correspondendo ao valor 1 do multiplicador.

A atividade desenvolvida pelos funcionários da empresa tem duração diária de 7 horas, o que corresponde ao valor 1 para o multiplicador.

$\mathrm{O}$ valor do SI calculado a partir dos valores dos multiplicadores referentes às variáveis da tarefa da retirada da cabeça do camarão foi igual a 9 para o punho direito e esquerdo. Esse valor é classificado como uma atividade arriscada para desenvolver distúrbios osteomusculares nos punhos. A aplicação do método SI permitiu constatar ainda o nível de risco biomecânico da atividade e a necessidade de intervenção ergonômica.

\section{Discussão}

O SI, como mencionado, é um método baseado na avaliação da interação multiplicativa entre seis variáveis da tarefa. A esse respeito, é similar em conceito ao limite de peso recomendado pelo Guia do Manual NIOSH. Alguns estudos sugerem ser coerente o conceito de interações multiplicativas entre as variáveis da tarefa e o risco de morbidade na extremidade distal superior (18-20).

A força excessiva é considerada um dos principais fatores de risco biomecânico determinantes de distúrbios musculoesqueléticos relacionados ao trabalho (21). Há associação estatisticamente significativa entre a ocorrência de tendinites e uso de força excessiva e alta repetitividade no trabalho, comparando-se com baixo emprego de força e pouca repetitividade no desempenho das tarefas (19). Contudo, após analisar a atividade, observou-se que os trabalhadores realizam a tarefa tranquilamente, e esta foi classificada como uma atividade leve, não causando sobrecarga 
biomecânica nos punhos e não sendo determinante para elevar a pontuação do SI.

A duração do esforço não tem somente implicações biomecânicas e fisiológicas, é na verdade um dos dois fatores de risco para a incidência total da taxa de morbidade dos punhos em um estudo epidemiológico (20).

Observou-se ausência de pausas para recuperação das estruturas teciduais durante a execução da atividade, visto que a duração do esforço no ciclo de trabalho foi igual ao tempo de ciclo de 1,7 segundos, necessário para a realização da retirada da cabeça do camarão. Quanto maior o tempo de esforço num determinado ciclo, menor o tempo que o trabalhador tem de descanso para se recuperar e a pausa durante o trabalho é uma indispensável condição fisiológica no interesse de manutenção da capacidade de produção (22). A fadiga e tempo de recuperação insuficiente podem causar lesões nos tendões (21). Assim, segundo o método utilizado, esse fator sobrecarrega biomecanicamente os punhos, elevando o valor da pontuação do SI.

Os movimentos de alta repetitividade durante o desenvolvimento da tarefa também são considerados uma das causas principais do aparecimento dos distúrbios osteomusculares. Nesse contexto, consideram-se como movimentos de alta repetitividade aqueles que possuem um ciclo básico de menos de 30 segundos e/ou atividades em que mais do que $50 \%$ do ciclo de trabalho envolve movimentos similares das extremidades superiores (23). Movimentos de punho altamente repetitivos durante o trabalho foram encontrados como estando diretamente associados à dor nessa região quando comparados ao trabalho com baixa repetitividade: prevalência de $56 \%$ para frequência de 31 esforços por minuto e prevalência de $26 \%$ para frequência de 16 esforços por minuto (24). Atividades altamente repetitivas podem lesar diretamente os tendões por meio das contrações e alongamentos repetidos (21). A frequência do esforço encontrada na retirada da cabeça do camarão foi de 35 esforços por minuto para ambos os punhos, sendo um item que sobrecarrega as estruturas musculoesqueléticas aumentando a pontuação do SI.

Realizou-se estudo (22) em que foram avaliados os efeitos do uso de um alicate, cuja forma foi modificada para se adaptar ao punho, e de um alicate normal, o qual exige desvio ulnar do punho para a sua utilização. Após 12 semanas de trabalho, 25 dos 40 colaboradores que tiveram que adotar a postura de desvio ulnar do punho apresentaram inflamações dos tendões dos músculos do antebraço na região do punho; em contrapartida, no grupo dos trabalhadores com uma postura neutra, com o alicate modificado, ocorreram somente 4 casos de inflamações de tendões dos músculos do antebraço na região do punho (22). Posturas articulares inadequadas (grande desvio ulnar, hiperextensão do punho) são fatores de riscos para o desenvolvimento de lesões musculoesqueléticas relacionadas ao trabalho nas mãos e punhos (25). Na presente pesquisa, a atividade de retirada da cabeça do camarão apresentou posturas de mãos-punhos direito e esquerdo classificadas como muito boa e boa, respectivamente. Assim, segundo o método Strain Index, a atividade não causa sobrecarga biomecânica e não é determinante na pontuação.

Vários estudos identificam a relação entre trabalho em ritmo elevado e distúrbios osteomusculares. Comparando duas indústrias produtoras de vidro em diferentes fases de incorporação tecnológica, foi encontrado um maior número de queixas de dores musculares na indústria em que o ritmo de trabalho é mais elevado (26). Existem evidências da relação entre risco de distúrbios osteomusculares no punho e a velocidade do esforço (27). No entanto, a velocidade de trabalho encontrada foi classificada como razoável para os punhos, ou seja, o funcionário executa a tarefa numa velocidade normal do movimento, que segundo o SI não sobrecarrega biomecanicamente as estruturas osteomusculares a ponto de causar lesão, não elevando o valor da pontuação.

0 excesso de horas de trabalho pode levar os colaboradores a excessiva fadiga física e mental, predispondo-os a distúrbios musculoesqueléticos e psicológicos relacionados ao estresse (28). No posto de trabalho avaliado, a duração da tarefa por dia foi de até 8 horas diárias. Jornadas de trabalho inferiores a 8 horas mantêm uma boa capacidade de produção dos trabalhadores, provoca um cansaço suportável e permite a recuperação da fadiga para o dia seguinte de trabalho $(22,29)$. Dessa forma, segundo o método Strain Index, a jornada dos trabalhadores avaliados não aumenta a carga biomecânica sobre o punho e não é um fator que eleva a pontuação do método.

Levando em consideração todos os seis itens da tarefa, obteve-se o valor de SI $=9$ para a retirada da cabeça do camarão, o que é interpretado como uma atividade arriscada para desenvolver distúrbios osteomusculares em ambos os punhos. A grande duração do esforço e alta repetitividade foram as principais causas que determinaram o valor elevado do SI.

Embora o objetivo deste trabalho tenha sido cumprido, reconhecem-se algumas limitações. Três dos 
seis itens avaliados (intensidade do esforço, postura e velocidade de esforço) têm avaliações qualitativas e contam com o julgamento subjetivo do pesquisador. Em particular, a intensidade do esforço tem um grande impacto sobre a pontuação do SI, mas a sua classificação é atribuída subjetivamente. Desse modo, as diferenças nas classificações podem advir de erros de avaliação, inexperiência, e/ou mau juízo. Esse é provavelmente um dos pontos fracos do Strain Index.

\section{Conclusão}

A indicação da presença dos riscos de distúrbios osteomusculares nos punhos durante a realização da atividade de trabalho por esses trabalhadores foi determinada pela pontuação do Strain Index, apontando para a necessidade de intervenção ergonômica. Esses distúrbios podem levar a diversos níveis de incapacidade, sendo também responsáveis por gastos com afastamentos, processos trabalhistas, tratamentos e processos de retorno e substituição no trabalho. 0 foco das empresas não deve ser apenas a produtividade e os lucros, mas também a saúde dos seus funcionários.

\section{Referências}

1. Candeias NMF. Conceitos de educação e de promoção em saúde: mudanças individuais e mudanças organizacionais. Rev Saúde Públ. 1997;31(2):209-13.

2. Maeno M. Reestruturação capitalista; [acesso 15 jan 2010]. Disponível em: http://www.fazer.com.br/ layouts/abrat/default2.asp?cod_materia $=2484$

3. Guimarães BM, Martins LB, de Azevedo LS, Andrade MA. Análise da carga de trabalho de analistas de sistemas e dos distúrbios osteomusculares. Fisioter Mov. 2011;24(1):115-24.

4. Ministério da Previdência e Assistência Social (Brasil), Instituto Nacional de Seguridade Social - INSS, Divisão de Planejamento e Estudos Estratégicos. Boletim Estatístico de Acidentes de Trabalho-BEAT; [acesso 20 mar 2007]. Disponível em: http://www. mpas.gov.br/pg_secundarias/paginas_perfis/perfil_Empregador_10_04-A5.asp

5. Fredrikson K. On causes of neck and shoulder pain in the general population [tese de doutorado]. Stockholm: National Institute for Working Life, 2000.
6. Balogh I. Questionnaire-based mechanical exposure indices for large population studies: reliability, internal consistency and predictive validity. Scand J Work Environ Health. 2001;27(1):41-48.

7. Chiang HC, Ko YC, Chen SS, Yu HS, Wu TN, Chang PY. Prevalence of shoulder and upper-limb disorders among workers in the fish-processing industry. Scand J Work Environ Health. 1993;19(2):126-31.

8. Kumar S. Theories of musculoskeletal injury causation. Ergonomics. 2001;44(1):17-47.

9. Coury HJCG, Walsh IAP, Pereira ECL, Manfrim GM, Perez L. Indivíduos portadores de L.E.R. acometidos há 5 anos ou mais: um estudo da evolução da lesão. Rev Bras Fisioter. 1999;3(2):79-86.

10. Barbosa LH, et al. Posturas corporais como indicador de risco músculo-esquelético em setores ocupacionais. In: Anais do Congresso Brasileiro de Ergonomia, 10., 2000; Rio de Janeiro.

11. Barbosa LH, Sturion HC, Walsh IAP, Alem MER, Coury HJCG. Abordagem da fisioterapia na avaliação de melhorias ergonômicas de um setor industrial. Rev Bras Fisioter. 2000;4(2):83-92.

12. Baldan C, Rodrigues JS, Nakano K, Walsh IAP, Alem MER, Coury HJCG. Avaliação dos aspectos pessoais ocupacionais e psico-sociais e sua relação no surgimento e/ou agravamento de lesões musculoesqueléticas em um setor de trabalho. Fisioter Mov. 2001;14(2):37-42.

13. Settimi MM, Toledo FF, Paparelli R, Martins M, Souza IM, Pinheiro JA. Lesões por esforços repetitivos (LER) e distúrbios osteomusculares relacionados ao trabalho (DORT). Campinas: Centro de Estudos em Saúde e Trabalho; 2000; [acesso 6 jul 2007]. Disponível em: www.ergonet.com.br/download_2/contrib-estudo-lerdort.pdf

14. Oliveira CR, organizador. Manual Prático de L.E.R. - Lesões por Esforços Repetitivos. Belo Horizonte: Editora Health; 1998.

15. Signori LU, Martins, MAP, Antonelo N, Godoy LP. Gerenciamento das LER/DORT através da organização do trabalho. Anais do ENEJEP - Encontro Nacional de Engenharia de Produção, 19.; 1999; Rio de Janeiro.

16. Moore JS, Garg A. The strain index: a proposed method to analyze jobs for risk of distal upper extremity disorders. Am Ind Hyg Assoc J. 1995;56(5):443-58. 
17. Borg G. Psychophysical scaling with applications in physical work and the perception of exertion. Scand J Work Environ Health. 1979;16(1):55-8.

18. Silverstein BA, Fine LJ. Armstrong TJ. Occupational factors and carpal tunnel syndrome. Am J Ind Med. 1987;11(3):343-58.

19. Armstrong TJ, Fine LJ, Goldstein SA, Lifshitz YR, Silverstein BA. Ergonomics considerations in hand and wrist tendonitis. J Hand Surg Am. 1987;12[5pt2]:830-7.

20. Moore JS, Garg A. Upper extremity disorders in a pork processing plant: relationships between job risk factors and morbidity. Am Ind Hyg Assoc J. 1994;55:703-15.

21. Keyserling WM, Stetson DS, Silverstein BA, Brouver ML. A checklist of evaluation risk factors associated with upper extremity cumulative trauma disorders. Ergonomics. 1993;36(7):807-31.

22. Grandjean E, Kroemer KHE. Manual de ergonomia: adaptando o trabalho ao homem. 5. ed. Porto Alegre: Bookman; 2005.

23. Maciel, RH. Ergonomia e lesões por esforços repetitivos (LER). In: Codo W, Almeida M. L.E.R. Lesões por esforços repetitivos, organizadores. 4. ed. Petrópolis: Vozes; 1995.
24. Hansson GA, Balogh I, Ohlsson K, Palsson B, Rylander L, Skerfving S. Impact of physical exposure on neck and upper limb disorders in female workers. Appl Ergon. 2000;31(3):301-10.

25. Chaffin DB, Anderson GBJ, Martin BJ. Biomecânica Ocupacional. Belo Horizonte: Ergo Editora; 2001.

26. Queiroz MF, Maciel RH. Condições de trabalho e automação: o caso do soprador da indústria vidreira. Rev Saúde Públ. 2001;35(1):1-9.

27. Marras WS. Schoenmarklin RW. Wrist motions in industry. Ergonomics. 1993;36(4):341-51.

28. Merlo ARC. A informática no Brasil: prazer e sofrimento no trabalho. Porto Alegre: Universidade UFRGS; 1999.

29. Lin TY, Teixeira MJ, Romano MA, Picarelli H, Settimi MM, Greve JMD’A. Distúrbios osteomusculares relacionados ao trabalho. Rev Med. 2001;80(ed. esp. pt. 2):422-42.

Recebido: 16/06/2012

Received: 06/16/2012

Aprovado: 17/10/2012

Approved: 10/17/2012 